ISSN 2447-9071

doi https://doi.org/10.36414/rbmc. v5i13.18

Contato para correspondência: Hermínio Maurício da Rocha Sobrinho

E-mail:

herminio.sobrinho@gmail.com

Conflito de interesse: Não

Financiamento: Recursos próprios

Recebido: 03/11/2019

Aprovado: 03/12/2019

\section{Acne vulgar: associações terapêuticas estéticas e farmacológicas}

\section{Acne vulgaris: aesthetic and pharmacological therapeutic associations}

\author{
Jéssica Gomide Pereira'; Kleber França Costa²; Hermínio Maurício da Rocha Sobrinho1,2 \\ 1 Pontifícia Universidade Católica de Goiás - PUC Goiás \\ ${ }^{2}$ Universidade Estadual de Goiás - UEG
}

\section{Resumo}

A acne vulgar é a afecção cutânea mais frequente na população mundial. Consiste em uma doença crônica da unidade pilossebácea com importantes repercussões psicossociais. Este estudo objetivou revisar as principais modalidades terapêuticas para o tratamento da acne vulgar, ressaltando-se os benefícios e a eficácia das terapias farmacológicas e estéticas nas diferentes formas clínicas da acne. Trata-se de uma revisão narrativa de literatura sobre a associação de terapias farmacológicase estéticas no tratamento da acne vulgar. Para a pesquisa foram utilizadas as bases de dados PubMed e Scielo. A busca das publicações foi realizada no período de Julho do ano de 2017 a Julho do ano de 2018. Foram selecionados artigos publicados de 2009 a 2018, com amostra final de 37 artigos. A classificação da acne vulgar quanto à gravidade das lesões em leve (comedoniana ou papulo-pustulosa), moderada (papulo-pustulosa ou nodular) e grave (nódulo-cística ou conglobata) mostra-se útil na determinação da terapêutica a ser escolhida, sendo adotada nos algoritmos terapêuticos de consensos internacionais. Os fármacos incluem retinoides tópicos e sistêmicos, antimicrobianos tópicos e sistêmicos, peróxido de benzoila, ácido azelaico, além de fototerapia e procedimentos estéticos. $O$ tratamento visa proporcionar a resolução das lesões cutâneas da acne, prevenir o desenvolvimento de cicatrizes e reduzir o aparecimento de novas lesões. Algumas terapias são recentes e promissoras, como éo caso de abordagens estéticas utilizando-se o laser, peeling químicos, microagulhamento e microdermoabrasão que podem colaborar como tratamento coadjuvanteà terapiamedicamentosa podendo apresentar efeitos sinérgicos no tratamento da acne leve a moderada.

Palavras-Chave: Acne vulgar, Tratamento medicamentoso, Terapia estética, Terapia combinada, Laserterapia.

\begin{abstract}
Acne vulgaris is the most common skin disorder in the world population. It is a chronic disease of the pilosebaceous unit with important psychosocial repercussions. This study aimed to review the main therapeutic modalities for the treatment of acne vulgaris, highlighting the benefits and efficacy of pharmacological and aesthetic therapies in different clinical forms of acne. This is a narrativeliterature review on the association of pharmacological and aesthetic therapies in the treatment of acnevulgaris. For the research we used the PubMed and Scielo databases. The search for publications was carried out from July 2017 to July 2018. Articles published from 2009 to 2018 were selected, with a final sample of 37 articles. The classification of acne vulgaris according to the severity of the lesions in mild (comedonian orpapulopustular), moderate (papulopustularornodular) and severe (nodularcysticorconglobata) is useful in determining the treatment to be chosen. adopted in the therapeutic algorithms of international consensus. Drugs include topical and systemic retinoids, topical and systemic antimicrobials, benzoyl peroxide, azelaic acid, phototherapy and aesthetic procedures. The treatmentaims to resolve acne skin lesions, prevent the development of scars and reduce the appearance of new lesions. Some therapies are recent and promising, such as aesthetic approaches using laser, chemical peels, micro-needling and microdermabrasion that may collaborate with the adjuvant treatment to drug therapy and may have synergistic effects in the treatment of mild to moderate acne.
\end{abstract}

Keywords: Acne vulgaris, Pharmacological treatment, Aesthetics therapy, Combined therapy, Laser therapy. 


\section{Introdução}

A acne é a doença dermatológica mais comum no mundo, acometendo milhares de pessoas. Em sua etiopatogenia, com frequência há evolução de lesões comedonianas para lesões inflamatórias das glândulas sebáceas e dos folículos pilosos, levando aos diferentes níveis de acometimento dessa afecção. É uma doença crônica que acomete, especialmente, a face, o dorso e o tórax. Embora não ofereça riscos à vida, pode levar à formação de cicatrizes inestéticas importantes na face, apresentando significantes repercussões psicossociais e prejuízo na qualidade de vida dos indivíduos afetados ${ }^{1,2,3}$.

As lesões acneicas sofrem influência hormonal, principalmente androgênios (testosterona). Geralmente se inicia na adolescência: pico de incidência dos 14 e 17 anos no sexo feminino, e 16 a 19 anos no masculino. Quatros fatores etiopatogênicos são relacionados ao desenvolvimento das lesões da acne vulgar: a hiperplasia da glândula sebácea, as modificações da queratinização folicular, a colonização da unidade pilossebácea por Propionibacterium acnes, Staphylococcus epidermidis e Malassezia furfur e a ação de fatores mediadores da inflamação. Tanto a hiperplasia quanto as modificações da queratinização folicular são relevantes para o surgimento das lesões não inflamatórias: os comedões - considerados a base para o aparecimento de lesões inflamatórias. Essa afecção possui relevantes repercussões sociais (desemprego), físicas (cicatrizes) e psicológicas (depressão, ansiedade, inibição social e baixa autoestima), que podem ser consideradas maiores e/ou mais negativas que outras doenças como epilepsia e asma ${ }^{2,4}$.

A acne vulgar é classificada, clinicamente, em: comedoniana, papulo-pustulosa, nodular cística e acne conglobata. Conforme a gravidade, acne é estadiada em leve, moderada ou grave. A acne comedoniana compreende lesões não inflamatórias envolvendo comedões abertos (conhecidos como cravos) e comedões fechados, com seborreia presente. Na acne papulo-pustular são encontradas lesões inflamatórias e não inflamatórias. As lesões inflamatórias superficiais são compostas de pápulas e pústulas. Lesões não inflamatórias constituem os comedões abertos e fechados. Na acne nodular são observados os nódulos que são lesões de consistência firme, dolorosas à palpação, com características inflamatórias e com diâmetro acima de $10 \mathrm{~mm}$. A acne conglobata consiste numa forma grave e rara da acne, mas sem repercussões sistêmicas na maioria das vezes. Nela são encontrados múltiplos comedões agrupados em meio à pápulas inflamatórias, além de nódulos supurativos dolorosos que coalescem para formar tratos sinusais. Além disso, são frequentes cicatrizes cutâneas extensas e desfigurantes ${ }^{5,6}$.

O tratamento da acne é variável conforme o grau de acometimento. São muitas as opções terapêuticas para essa afecção cutânea, podendo ser empregadas terapias de modo isolado ou em combinação. $O$ tratamento farmacológico é aplicado sob a forma tópica e/ou sistêmica, de forma a controlar o avanço e evitar sequelas que podem ser mais preocupantes num segundo momento. A maior parte dos tratamentos estéticos apresentam finalidade comedolítica, reduzem a seborreia e a inflamação e ainda eliminam ou melhoram a aparência das cicatrizes de acne ${ }^{5,6}$.

O presente estudo teve como objetivo revisar as principais modalidades terapêuticas para o tratamento da acne vulgar, ressaltando-se os benefícios e a eficácia das terapias farmacológicas e estéticas nas diferentes formas clínicas da acne.

\section{Métodos}

Este trabalho trata-se de uma revisão narrativa de literatura sobre a associação de terapias farmacológicas e estéticas no tratamento da acne vulgar. Para a pesquisa foram utilizadas as bases de dados eletrônicas: Pubmed (US National Library of Medicine) e Scielo (Scientific Eletronic Library Online). A busca das publicações foi realizada no período de Julho do ano de 2017 a Julho do ano de 2018, com o uso dos Descritores em Ciências da Saúde (DeCS) e palavras-chave: acne vulgaris, treatment acne, combination therapy, acid acne, aesthetic therapy acne, lasers e acne. Foram selecionados artigos publicados nos idiomas inglês, português e espanhol, no período de 2009 a 2018, que apresentavam conteúdo completo disponível, tendo sido encontrados 692 artigos sobre o tratamento de acne vulgar. Do material pesquisado encontrado, foram selecionados 37 artigos que apresentavam conteúdos que, realmente, contribuíram para o cumprimento dos objetivos do presente trabalho. Foram excluídos trabalhos cujos conteúdos não estavam relacionados aos objetivos do presente estudo e fora do período temporal determinado.

\section{Resultados e Discussão}

Na abordagem terapêutica da acne vulgar deve-se realizar, além da imprescindível abordagem dermatoscópica da pele, uma anamnese minuciosa e exame sobre o estado clínico do paciente, comorbidades, antecedentes patológicos, estado emocional e histórica de alergias. Além disso, quando do uso da isotretinoína sistêmica, deve-se fazer uma avaliação laboratorial, solicitando-se beta-HCG, hemograma, perfil lipídico, exames hepáticos e de função renal, e outros que a avaliação clínica indicar necessários ${ }^{5-7}$.

\section{1) Abordagens Estéticas e de Terapias Complementares}

O tratamento atual da acne vulgar inclui o uso adjuvante de procedimentos estéticos tais como os peelings químicos, micro- 
dermoabrasão, o microagulhamento e a fototerapia (lasers) com a terapia farmacológica tradicional7.

\section{Limpeza de pele}

A higienização cutânea é um relevante cuidado inicial à acne vulgar, sendo sempre eficiente, exceto se exagerada, e visa à redução da seborreia. As formulações desenvolvidas com benefícios contra acne ativa podem apresentar ácido salicílico, taninos, hamamélias, óleo de melaleuca, eucalipto e a-hidroxiácidos8.

\section{Peelings químicos}

O peeling químico é um procedimento amplamente utilizado no tratamento de acne vulgar ativa e de suas cicatrizes. Os agentes mais utilizados são: o ácido salicílico, o ácido glicólico, o ácido pirúvico, o ácido retinoico, o ácido láctico, o ácido mandélico, a solução de Jessner, o ácido tricloroacético e o fenol. O peeling adequado é escolhido com base no fototipo cutâneo, a partir da classificação de Fitzpatrick, na atividade da acne e no tipo de cicatrizes de acne, sendo que tem, principalmente, efeito comedolítico e colabora na resolução de lesões não inflamatórias. Os peelings combinados minimizam os efeitos colaterais. Em cicatrizes de acne, peelings químicos podem ser combinados com outros procedimentos para alcançar melhores resultados clínicos ${ }^{8,9}$.

O Ácido Salicílico e a Resorcina são úteis no tratamento da acne ativa leve, visto serem queratolíticos e esfoliantes. Os efeitos colaterais mais comuns são eritema, ressecamento da pele, queimaduras e hiperpigmentação temporária. O peeling de ácido glicólico mostrou melhora sobre pápulas e pústulas. Como consequência de peelings de ácido glicólico está a hiperpigmentação da pele $e^{9,10,11,12}$.

Outro agente de descamação química é o ácido tricloroacético (ATA). Esse pode ser aplicado sozinho ou em combinação com outros agentes químicos. Como efeitos colaterais, há o risco de hipopigmentação ou hiperpigmentação pós-inflamatória, que pode ser observada mais comumente em concentrações mais elevadas e em pacientes com fototipos V e VI ou melanodérmicos ${ }^{9,10,12}$.

\section{Dispositivos de Luz e Laserterapia}

De modo geral, supõe-se que a luz atue provocando efeitos anti-inflamatórios e bactericidas, com diminuição da produção e liberação de citocinas, além de efeitos como o aumento da atividade da glândula sebácea e a formação de espécies reativas de oxigênio $0^{4,13,14}$.

Os lasers, em particular, também têm um papel central no tratamento da cicatrização da acne, que tende a ser refratária às terapias médicas. A fototermólise fracionada e o laser de diodo de $1450 \mathrm{~nm}$ têm sido utilizados no tratamento não-ablativo de cicatrizes de acne com sucesso significativo. A Luz Intensa Pulsada
(LIP) tem um papel relevante na acne vulgar ativa, porém tem seu uso contraindicado quando paciente estiver terapia com Isotretinoína ${ }^{7,15}$.

\section{Uso de Diodos Emissores de Luz (LEDs)}

$\mathrm{O}$ uso de LEDs com frequências individualizadas, como a de $415 \mathrm{~nm}$ (azul), $633 \mathrm{~nm}$ (vermelho) e $830 \mathrm{~nm}$ (infravermelho), demonstrou resultados significativos para o tratamento de acne vulgar leve a moderada ${ }^{13}$.Eventos adversos, efeitos colaterais locais e sistêmicos são $\operatorname{raros}^{7,13,14}$.

O alvo da terapia de luz para acne vulgar depende em parte das características dabactéria $P$. acnes, em que as porfirinas produzidas pelas bactérias absorvem luz no espectro visível. A mais abundante é a coproporfirina III, que tem pico de absorção em $415 \mathrm{~nm}$, e as espécies reativas de oxigênio produzidas por fotoexcitação podem eliminar o $P$. acnes $^{14}$.

\section{Laser de diodo de 1.450nm}

O laser de diodo de 1.450nm tem sido utilizado para o tratamento de acne vulgar e de cicatrizes de acne. Este laser tem sido considerado uma terapia adjuvante para o tratamento de acne moderada a grave, com remissão a longo prazo. A melhora clínica pode ser mantida por até doze meses, sendo bem mais significativo quando se compara com tratamento tópicos ou com o uso de antibióticos ${ }^{16}$.

\section{Laser de fototermólise fracionado}

Em 2003 foi criada a tecnologia de laser de fototermólise fracionado para superar as limitações dos tradicionais lasers. A diferença é que o laser de fototermólise age na microscopia criando zonas de danos térmicos em que é possível a separação espacial entre os tecidos danificados, chamados de zonas microtérmicas e a pele saudável ${ }^{17}$.

Como efeito adverso ao uso do laser fracionado de fototermólise os pacientes podem apresentar: eritema, hiperpigmentação pós-inflamatória e desconforto ${ }^{17}$.

\section{Laser fracionado com radiofrequência fragmentada}

A combinação de um laser fracionado com um dispositivo de radiofrequência fragmentadatem como vantagem o fato de poder ser usado em todos os tipos de pele. Como efeito adverso, a presença da hiperpigmentação pós-inflamação pode variar de acordo com o tipo de laser utilizado ${ }^{18}$.

\section{Laser Erbium fracionado não ablativo ProDeep 1340nm}

O Laser Prodeep tem sido utilizado na terapêutica de cicatrizes de acne vulgar. Esse tipo de Laser tem se mostrado bastante efetivo e seguro. Seu principal modo de ação deve-se ao estímulo a 
síntese de colágeno. Além disso, a associação do Laser Prodeep com microagulhamento em múltiplas sessões desse tratamento (4 a 6), tem sido recomendada para se alcançar a melhor eficáciaa ${ }^{17,18}$.

\section{Laser fracionado de granito de alumínio de ítrio-érbio}

O uso de laser ablativos tem mostrado melhoras significativas, no entanto, os efeitos adversos como despigmentação e eritema prolongado pós procedimento têm feito seu uso ser reduzido, principalmente em pacientes com pele mais escura. Por outro lado, o laser não-ablativo como o diodo, granito de alumínio de ítrio com neodímio ( $\mathrm{Nd}$ : YAG) e laser pulsado têm apresentado mais segurança com baixa eficácia ${ }^{17}$.

O laser fracionado de revestimento Er:YAG 2940nm melhora efetivamente a cicatriz acneica em um curto espaço de tempo e com baixo risco de reações adversas ${ }^{17}$.

\section{Microdermoabrasão}

A microdermoabrasão é uma técnica popular usada no tratamento de diferentes afecçõesda pele, incluindo acne, cicatrizes de acne, estrias de distensão e fotoenvelhecimento ${ }^{4,19}$. Nessa técnica, a abrasão é condicionada aos seguintes aspectos: quantidade de microcristais jateados, pressão utilizada e número procedimentos realizados sobre a pele tratada. Ela resulta em melhoria das irregularidades do contorno da pele, possibilita o nivelamento tecidual, pela esfoliação de suas extremidades, além de estímulo para um incremento proteico adequado na região depressiva. Seu papel no tratamento de lesões e cicatrizes de acne vulgar é limitado ${ }^{19,20}$.

\section{Microagulhamento}

No microagulhamento, ou terapia de indução de colágeno, as microagulhas levam a microtraumas da derme superficial. Nesses locais de microtraumas dérmicos podem ser adicionados agentes, como fatores de crescimento, que estimulam a neocolagênese. Isso provoca a formação de um novo tecido conjuntivo que preenche regiões de cicatrizes atróficas, além de induzir a repigmentação do local, através da melhoria do suprimento de sangue para a região tratada. O remodelamento do tecido tratado persiste por meses após realização do procedimento $21,22,23,24$.

Como efeitos adversos mínimos esse procedimento pode apresentar: dor leve, eritema pós-procedimento, pequenos hematomas e edema. Não têm sido relatadas complicações relacionadas à infecção $21,26,27$.

\section{Terapias complementares para acne vulgar}

Novas abordagens da Medicina Complementar e Alternativa, como a acupuntura, fitoterapia, uso de probióticos e modificações dietéticas, vem ganhando mais notoriedade devido às preocu- pações dos pacientes sobre os efeitos adversos de tratamentos convencionais para a acne vulgar ${ }^{28}$.

Os fitoterápicos mais descritos recentemente com melhores eficácias são a epigalocatequina galato (EGCG) e o resveratrol. O EGCG, é um fitoquímico derivado do chá verde, que de acordo com alguns estudos pode ter ações anti-inflamatórias e ainda suprimir a produção do sebo, provocando poucos efeitos colaterais como, eritema e irritação local leve. Já o resveratrol, pode ser encontrado em uvas e amendoins com as mesmas atividades do EGCG, no entanto sem relatos de efeitos colaterais até o momento ${ }^{4}$.

Os probióticos têm sido opção como imunomodulador em doenças inflamatórias como a ance. O mecanismo de ação dos probióticos está diretamente relacionado à reintrodução das bactérias (Nitrosomonas eutropha) originalmente presentes na pele humana, onde auxiliavam a resposta inflamatória local, provocando melhora das lesões acneicas 4 .

\section{2) Tratamento Farmacológico e Terapias Combinadas na Acne Vulgar}

\section{Tratamento da acne comedoniana}

Para o tratamento da acne comedoniana, a melhor eficácia foi demonstrada com o uso de retinóides tópicos (RTs): adapaleno, tretinoína e isotretinoína (grau médio de recomendação pelo European Guideline)6. O uso de RTs favorece a redução de microcomedões. $\mathrm{O}$ adapaleno é o retinoide com menor potencial irritante, quando aplicado pela manhã, porém os demais RTs podem ter os seguintes efeitos adversos: xerose, irritação, intolerância solar (quando não aplicados a noite). Como alternativas terapêuticas podem-se adotar o ácido azelaico (AA), o peróxido de benzoíla (PBO), porém com baixa força de recomendação ${ }^{29}$.

Comparando-se a eficácia de ativos no tratamento de lesões da acne comedônica, percebe-se que a associação de adapaleno e PBO mostra uma melhor eficácia do que quando comparada ao uso isolado de PBO, porém equiparável ao uso de adapaleno. Apesar disso, essa combinação demonstrou uma melhor tolerabilidade dos ativos pelo paciente ${ }^{6,30}$.

Lasers de onda visíveis ou de onda infravermelhos, luz intensa pulsada e fotodinâmica são opções terapêuticas que necessitam de maiores estudos que certifiquem a efetividade dessas técnicas para o tratamento da acne comedônica. Não são indicados para esse tipo de acne: antibióticos tópicos, terapia hormonal antiandrogênica, antibióticos sistêmicos e isotretinoína sistêmica, nem o uso de radiação ultravioleta artificial $^{6}$.

\section{Tratamento da acne papulo-pustular leve a moderada}

Conforme o European acne guidelines, a combinação de adapaleno e PBO é fortemente recomendada para o tratamento de 
acne papulo-pustular leve a moderada6. Também é fortemente recomendado o uso da combinação de PBO e Clindamicina para esse tipo de acne. Ainda segundo esse Guidelines, com força média de recomendação estão: o ácido azelaico, o peróxido de benzoíla, a combinação de antibiótico sistêmico com adapaleno, a associação de clindamicina e de tretinoína tópicas, além de retinoides tópicos ${ }^{31}$.

Outras opções, com baixa força de recomendação estão: monoterapia com luz azul; zinco oral; antibióticos sistêmicos em combinação com ácido azelaico; associação de antibiótico sistêmico com adapaleno e peróxido de benzoila; combinação de antibiótico sistêmico com peróxido de benzoíla; associação de eritromicina e isotretinoína; ou a combinação de eritromicina e tretinoína. Não são recomendados para esse tipo de lesão: monoterapia com antibióticos tópicos; radiação Ultravioleta (UV) artificial; combinação de eritromicina e zinco; terapia sistêmica com antiandrogênios, isotretinoína e antibióticos ${ }^{5,6,30}$.

\section{Tratamento da acne pápulo-pustular moderada a grave e acne nodular}

No tratamento da acne papulo-pustular moderada a grave ou da acne nodular, o tratamento sistêmico com isotretinoína é recomendado com base na excelente eficácia observada na prática clínica ${ }^{5,32}$.

Com força média de recomendação estão: o uso combinado de antibióticos sistêmicos com adapaleno, peróxido de benzoíla ou ácido azelaico. Com baixa força de recomendação está o uso combinado de antibiótico sistêmico com peróxido de benzoila. $\mathrm{Na}$ escolha dos antimicrobianos deve-se dar preferência pela Doxiciclina e Limeciclina, em detrimento das terapias anteriormente utilizadas (Minociclina e Tetraciclina). Antibióticos orais como monoterapia não são recomendados. O principal efeito da Doxiciclina é fotossensibilidade, e da Minociclina é a disfunção hepática. Uso de azitromicina tem sido relacionado com o surgimento de resistência bacteriana. Vale destacar que há um importante risco de insuficiência hepática pela associação da Tetraciclina e a Isotretinoína ${ }^{6,30}$.

Não há evidências suficientes para recomendar o tratamento dessas lesões com: monoterapia de antiandrogênios; monoterapia com luz visível; laser; fontes de luz que não a luz azul; fontes artificiais de radiação UV ${ }^{6,30}$.

Para mulheres em tratamento pode-se adotar a terapia hormonal antiandrogênica combinada a antibióticos sistêmicos e tópicos (incluindo antibióticos). Ou, ainda, pode-se fazer apenas a combinação dessa terapia hormonal com tratamentos tópicos (incluindo antibióticos) $)^{6,33,34}$.

\section{Tratamento de acne nodular grave e acne conglobata}

A Isotretinoína sistêmica é a terapia mais efetiva no tratamento da acne nodular grave e acne conglobata, em relação a outras opções como antibióticos sistêmicos ou monoterapia tópica. Desse modo, a isotretinoína oral é fortemente recomendada como monoterapia, tal como proposto pelo European Acne Guidelines ${ }^{5,6,32,35}$. Porém, a isotretinoína não dever ser usada na gestação, devido a sua alta teratogenicidade. Outros efeitos colaterais desse retinoide incluem: fotossensibilidade, queilite, xerose, epistaxe, alterações do perfil lipídico, da função hepática e de plaquetas.

De acordo com o European Acne Guidelines, em nível intermediário de recomendação para o tratamento desses tipos de acne estão: a combinação de antibióticos sistêmicos, adapaleno e peróxido de benzoila; a associação de antibióticos sistêmicos com ácido azelaico. Com baixo nível de recomedação podem ser considerados; o uso combinado de antibióticos sistêmicos e adapaleno; a associação de antibióticos sistêmicos com peróxido de benzoila. Embora a Terapia fotodinâmica seja eficaz no tratamento dessas lesões, ela ainda não pode ser recomendada devido à falta de regimes de tratamento padrão que assegurem protocolos com reações adversas agudas mínimas. Além disso não são recomendados: monoterapia com antibióticos sistêmicos; monoterapia com anti-andrógenos orais; Fontes artificiais de radiação UV; monoterapia com Luz visível ${ }^{36,37}$. Considerando-se o risco do uso de isotretinoína em mulheres em idade fértil, pode-se optar pela terapia hormonal antiandrogênica combinada a antibióticos sistêmicos e tópicos no tratamento de acne nodular grave e acne conglobata. Outra opção é o uso de antiandrogênios hormonais com tratamentos tópicos ${ }^{6,29,33,34}$ (Figura 1).

Figura 1. Fluxograma representativo da Terapêutica para Acne vulgar.

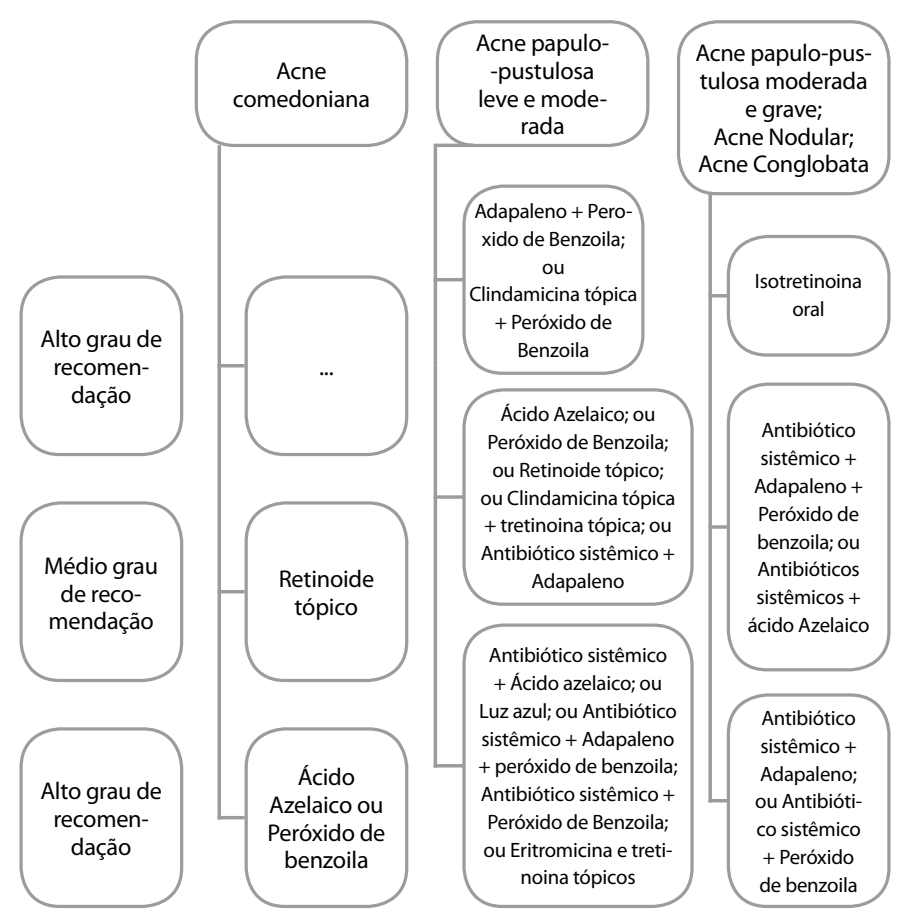


Em suma, há um conjunto de recomendações específicas para cada tipo de lesão acneica: comedônica, papulo-pustular leve a moderada, papulo-pustular grave, acne nodular, acne nodular grave e acne conglobata6,36. Vale destacar ainda que, embora a acne vulgar geralmente não oferece riscos à vida, essa doença apresenta importantes repercussões psicossociais, emocionais e de relacionamento interpessoal, o que merece uma atenção mais redobrada na avaliação dos diferentes tipos de lesões, bem como um cuidado mais especial para as peculiaridades das abordagens terapêuticas34,36,37,39.

\section{Acne na Gestação}

Com frequência há o aparecimento de lesões pustulosas acneicas durante a gravidez, mesmo naquelas que não possuía acne vulgar. Isso pode ser considerado normal, devido a elevação de síntese hormonal, com efeitos na hipersecreção sebácea. Para o tratamento da acne na gestação, recorrer ao uso da terapia tópica, como ácido azelaico e a limpeza de pele. Apesar disso, não são recomendados nesse período para o tratamento da acne: retinoides tópicos, peeling, antibioticoterapia, uso de lasers e radiofreqüência29,34,39. As diretrizes atuais com as recomendações para o tratamento da acne vulgar segundo a Sociedade Brasileira de Dermatologia estão representadas no fluxograma da figura 1.

\section{Conclusão}

$\mathrm{Na}$ abordagem terapêutica da acne vulgar, é fundamental que se possa delinear estratégias de tratamentos atualizados e apropriados conforme o aspecto clínico da lesão, bem como com as características individuais de cada caso. Ela visa proporcionar a resolução das lesões cutâneas, a prevenção do desenvolvimento de cicatrizes, sobretudo, oriundas de quadros graves dessa afecção, bem como a minimização do aparecimento de novas lesões básicas, como os comedões.

Algumas terapias são recentes e promissoras, como é o caso de abordagens estéticas como os peelings químicos, os Lasers, Leds, Microagulhamento e Microdermoabrasão que atuam de forma adjuvante no tratamento de lesões da acne vulgar podendo ser utilizadas junto com a terapia farmacológica.

As terapias mais utilizadas envolvem o uso de: retinoides tópicos, peróxido de benzoila, antimicrobianos tópicos ou sistêmicos, ácido azelaico e retinoides sistêmicos. O principal agente no tratamento das lesões moderadas a severas de acne vulgar é o uso de isotretinoina oral. Esse fármaco tem excelentes resultados, porém apresenta riscos relevantes de eventos adversos, se não observadas as devidas contraindicações de uso, sobretudo, em gestantes. Por isso, esta última abordagem deve ser conduzida por profissional dermatologista.

Vale destacar que os resultados de muitos tratamentos não são imediatos. Assim, as abordagens terapêuticas necessitam ser reavaliadas, frequentemente, para melhor condução clínica e adesão terapêutica do paciente, notadamente nos casos de acne resistente ao tratamento, ou quando há presença de cicatrizes importantes, ou então na suspeita de outras condições clínicas que estejam envolvidas no aparecimento de lesões similares a acne vulgar.

\section{Referências}

1. Bhate K, Williams HC. Epidemiology of acne vulgaris. Br J Dermatol. 2013;168: 474-85.

2. Figueiredo A, Massa A, Picoto A, Soares AP, Basto AS, Campos $C R$, et al. Avaliação e tratamento do doente com acne - Parte I: Epidemiologia, etiopatogenia, clínica, classificação, impacto psicossocial, mitos e realidades, diagnóstico diferencial e estudos complementares. Rev Port Clin Geral. 2011;27:59-65.

3. Silva AMF, Costa, FP, Moreira, M. Acne vulgar: diagnóstico e manejo pelo médico de família e comunidade. Rev Bras Med Fam Comunidade. 2014;9:54-63.

4. Trivedi, MK, Bosanac SS, Sivamani RK, Larsen LN. Emerging therapies for Acne Vulgaris. Am J Clin Dermatol. 2018;19(4):505-51. 5. Figueiredo A, Massa A, Picoto A, Soares AP, Basto AS, Campos $C R$, et al. Avaliação e tratamento do doente com acne - Parte II: Tratamento tópico, sistémico e cirúrgico, tratamento da acne na grávida, algoritmo terapêutico. Rev Port Clin Geral. 2011;27:66-76. 6. Nast A, Dréno B, Bettoli V, Bukvic Mokos Z, Degitz K, Dressler C, et al. European evidence-based: S3 guideline for the treatment of acne. European Dermatology Forum: subcommittee "Acne". JEADV. 2016;30:1-28.

7. Kim RH, Armstrong, AW. Current state of acne treatment: highlighting lasers, photodynamic therapy, and chemical peels. Dermatol Online J. 2011;15(17):1-2.

8. Peric S, Bubanj M, Bubanj S, Jancic S. Side effects assessment in glicolyc acid peelings in patients with acne type I. Bosn J Basic Med Sci. 2011;11(1):52-7.

9. Kontochristopoulos G, Platsidaki E. Chemical peels in active acne and acne scars. Clin Dermatol. 2017;35(2):179-82.

10. Al-Talib H, Al-Khateeb A, Hameed A, Murugaiah C. Efficacy and safety of superficial chemical peeling in treatment of active acne vulgaris. An Bras Dermatol. 2017;92(2):212-6.

11. Kaminaka C, Uede $M$, Matsunaka $H$, Furukawa $H$, Yamamoto $Y$. Clinical evaluation of glycolic acid chemical peeling in patients with acne vulgaris: a randomized, double-blind, placebo-controlled, split-face comparative study. Dermatol Surg. 2014;40(3):314-22.

12. Dréno B, Fischer TC, Perosino E, Poli F, Vieira MS, Rendon MI, et al. Expert opinion: efficacy of superficial chemical peels in active acne management - what can we learn from the literature 
today? Evidence-based recommendations. J Eur Acad Dermatol Venereol. 2011;25:695-704.

13. Ablon G. Phototherapy with Light Emitting Diodes: Treating a Broad Range of Medical and Aesthetic Conditions in Dermatology. J Clin Aesthet Dermatol. 2018;11(2):21-7.

14. Rai R, Natarajan K. Laser and light based treatments of acne: Review article. Indian J Dermatol Venereol Leprol. 2013;79(3):300-9. 15. Nestor MS, Swenson N, Macri A. Physical Modalities (Devices) in the Management of Acne. Dermatol Clin. 2016;34(2):215-23. 16. Alam M, Gladstone HB, Tung R. Dermatologia Cosmética: Requisitos em Dermatologia. Rio de Janeiro: Elsevier, 2011.

17. Nirmal B, Pai SB, Sripathi H, Rao R, Prabhu S, Kudur MH, et al. Efficacy and safety of Erbium-doped Yttrium Aluminium Garnet fractional resurfacing laser for treatment of facial acne scars. Indian J Dermatol Venereol Leprol. 2013;79:193-8.

18. Peterson JD, Palm MD, Kiripolski MG, Guiha IC, Goldman MP. Evaluation of the effect of fractional laser with radiofrequency and fractionated radiofrequency on the improvement of acne scars. Dermatol Surg. 2011;37:1260-7.

19. El-Domyati M, Hosam W, Abdel-Azim E, Abdel-Wahab $\mathrm{H}$, Mohamed E. Microdermabrasion: a clinical, histometric, and histopathologic study. J Cosmet Dermatol. 2016;15(4):503-13.

20. Karimpour DJ, Karimpour G, Orringer JS. Microdermabrasion: an evidence-based review. Plast Reconstr Surg. 2010;125(1):372-7. 21. Dogra S, Yadav S, Sarangal R. Microneedling for acne scars in Asian skin type: an effective low cost treatment modality. J Cosmet Dermatol. 2014;13:180-7.

22. Leheta T, Tawdy A. Percutaneous collagen induction versus full-concentration trichloroacetic acid in the treatment of atrophic acne scars. Dermatol Surg. 2011;37(2):207-16.

23. Liebhl H, Kloth LC. Skin cell proliferation stimulated by microneedles. J Am Coll Clin Wound Spec. 2012;4(1):2-6.

24. Sharad J. Combination of microneedling and glycolic acid peels for the treatment of acne scars in dark skin. J Cosmet Dermatol. 2011;10(4):317-23.

25. Rana S, Mendiratta V, Chander R. Efficacy of microneedling with $70 \%$ glycolic acid peel vs microneedling alone in treatment of atrophic acne scars-A randomized controlled trial. J Cosmet Dermatol. 2017;16(4):454-9.

26. Al-Qargaz F, Al-Yousef A. Skin microneedling for acne scars associated with pigmentation in patients with dark skin. J Cosmet Dermatol. 2018;17(3):390-5.

27. Kalil CLPV, Frainer RH, Dexheimer LS, Tonoli RE, Boff AL. Tratamento das cicatrizes de acne com a técnica de microagulhamento e drug delivery. Surg Cosmet Dermatol. 2015;7(2): 144-8.

28. Cao H, Yang G, Wang Y, Liu JP, Smith CA, Luo H, Liu Y. Complementary therapies for acne vulgaris. Cochrane Database Syst Rev. 2016;19(1):1-138.
29. Montagner S, Costa A. Diretrizes modernas no tratamento da acne vulgar: da abordagem inicial à manutenção dos benefícios clínicos. Surg Cosmet Dermatol. 2010;2(3):205-13.

30. Leyden J, Stein-Gold L, Weiss J. Why topical retinoids are mainstay of therapy for acne. DermatolTher (Heidelb). 2016;7(3):293304.

31. See JA, Goh CL, Hayashi N, Suh DH, Casintahan FA. Optimizing the use of topical retinoids in Asian acne patients. J Dermatol. 2018;45(5):522-8.

32. Costa CS, Bagatin E. Evidências sobre o tratamento da acne. Diagn Tratamento. 2013;18(1):10-4.

33. Addor FAZ, Schalka S. Acne da mulher adulta: aspectos epidemiológicos, diagnósticos e terapêuticos. An Bras Dermatol. 2010;85(6):789-95.

34. Ribeiro BM, Follador I, Costa A, Francesconi F, Neves JR, Almeida LMC. Acne da mulher adulta: revisão para o uso na prática clínica diária. Surg Cosmet Dermatol. 2015;7(3):10-9.

35. Bhat YJ, Latief I, Hassan I. Update on etiopathogenesis and treatment of Acne: review article. Indian J Dermatol Venereol Leprol. 2017;83(3):298-306.

36. Rzany B, Nast A. Acne treatment in the field: how guidelines and other sources can be included in daily practice. JEADV. 2013;27(2):2-5.

37. Campos V, Jordão J. Efficacy and patient satisfaction of a new nonablative fractional Nd:YAP laser $1340 \mathrm{~nm}$ for facial rejuvenation in Brazilian patients. Journal of the American Academy of Dermatology (JAAD). 2014;70(5-AB):199.

38. Hoffman-Cachafeiro T. Comparação entre laser erbium fracionado não ablativo 1340nm e microagulhamento para tratamento de cicatrizes atróficas de acne: ensaio clínico randomizado. Dissertação de Mestrado. Universidade Federal do Rio Grande do Sul, Faculdade de Medicina, Programa de pós-graduação em Medicina: Porto Alegre, 2015.

39. Souza CMD. O estresse biopsicossocial, qualidade de vida de jovens na cidade de Goiânia e infecções bacterianas e fúngicas dos tecidos queratinizados. Dissertação de Mestrado. Universidade Católica de Goiás, Programa de pós-graduação (Mestrado) em Ciências Ambientais e da Saúde: Goiânia-GO, 2004. 